\title{
ROBUSTNESS OF THE LIEBHERR-AEROSPACE EHA TECHNOLOGY FOR FUTURE FLIGHT CONTROL APPLICATION
}

\author{
Tobias Röben ${ }^{1 *}$, Emmanuel Viennet ${ }^{2}$, Henry Wider $^{3}$ \\ ${ }^{1}$ Liebherr-Aerospace Lindenberg GmbH, Pfänderstrasse 50-52, 88161 Lindenberg, Germany \\ ${ }^{2}$ HES-SO University of Applied Sciences and Arts Western Switzerland, 1700 Fribourg, Switzerland \\ ${ }^{3}$ Liebherr Machines Bulle SA, Rue Hans Liebherr 7, 1630 Bulle, Switzerland \\ * Corresponding author: Tel.: +49 838146 5615; E-mail address: tobias.roeben@liebherr.com
}

\begin{abstract}
Future more electric aircraft (MEA) architectures require a new generation of power-by-wire actuators, e.g. Electro Hydrostatic Actuators (EHA). These units have to be capable of frontline operation of safety critical flight control surfaces over the entire aircraft life. Prove of robustness becomes a challenging objective. This paper illustrates the Liebherr-Aerospace EHA technology as well as methods for validation of robustness. It gives an insight regarding the design of a robust piston pump.
\end{abstract}

Keywords: EHA, More Electric Aircraft, piston pump, robustness

\section{BACKGROUND}

State of the art aircraft architectures for civil aircraft embody a significant share of electrically driven systems (More Electric Aircraft) compared to the traditional importance of hydraulics and pneumatics in aviation. These more electric architectures require new equipment, e.g. power-by-wire actuators and power control electronics. For the safety critical application of primary flight controls ElectroHydrostatic Actuators (EHA) are favored, because they still rely on hydraulic actuation (refer to Figure 1). However, in contrast to conventional servo-hydraulic actuators these units form a local, independent hydraulic circuit, which is powered by an electric motor and a fixed displacement piston pump. Therefore, hydraulic power is generated only, when it is needed for the actuation of the relevant flight control surface. Furthermore, these units are easy to install and maintain.

Axial piston pumps are of great importance in industrial applications. Nevertheless, this experience cannot be transferred to aviation. While pumps are usually operated in a distinctive nominal condition, e.g. to maintain a certain pressure level, EHA for flight control application are designed to provide maximum performance under worst-case condition: low temperature, high air loads and dynamics.

However, the actual dynamic and loads are less most of the time. The pump is even operated bidirectional to enable extension/retraction and in motor mode, when external loads are supporting the motion. Thus, the pump has to provide good efficiency in all these operating conditions and it must especially be robust against operation in the partial load range.

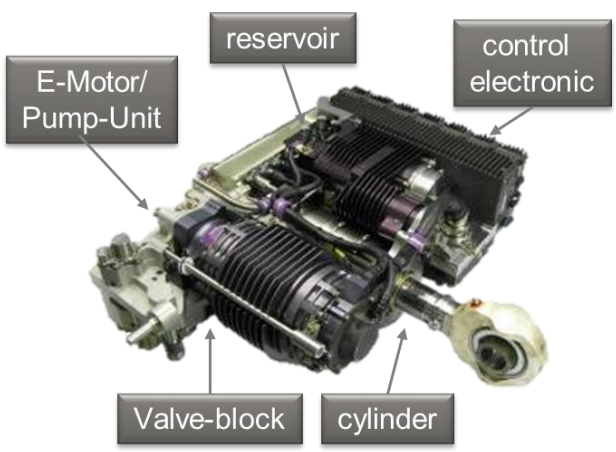

Figure 1: State of the art Liebherr-Aerospace EHA

\section{ENDURANCE TESTING}

Since stick-slip effects and mixed friction are critical mechanisms causing pump wear, the varying operating conditions must be considered, in order to prove the robustness of EHA for the safety critical flight control application. For this reason, Liebherr-Aerospace chose a two-way approach of testing. 


\subsection{Unit level tests}

To prove robustness in a most representative environment, a qualification test rig of an inseries project has been re-used for long term testing considering a representative endurance cycle (Figure 2). Here every test hour relates to one Flight Hour (FH), but in laboratory environment.

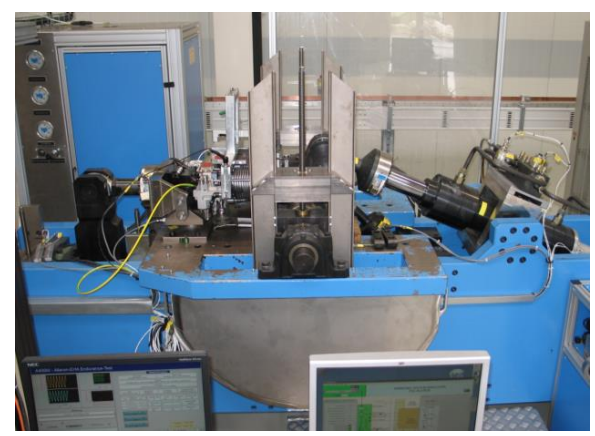

Figure 2: Unit level test endurance test rig

This approach is elaborative and expensive: One random sample unit was tested and operated for more than 5 years to accumulate about $35^{\prime} 000 \mathrm{FH}$. Since it constitutes merely one sample of a given application, it only gives an idea of the robustness of this technology. Nevertheless, such a test shows the general long-term behavior of the pump design.

Basically, three interfaces of tribological contact can be distinguished (refer to Figure 3):

- Cylinder barrel / valve plate (yellow)

- Piston / cylinder bore (blue)

- Slipper / swash plate (red)

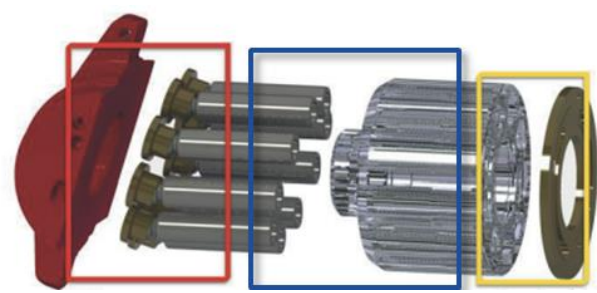

Figure 3: Interfaces of tribological contact within a fixed displacement axial piston pump [1]

Thanks to state-of-the-art manufacturing tolerances and surface treatments, usually the piston and cylinder bore interface is not critical in axial piston pumps [2]. The more sensitive interfaces are the valve plate interface and the swash plate interface.

To avoid jamming of contact pairs in motion usually a weaker and a more hard material is chosen. This combination inherently causes the weaker material to wear off during operation. For all three tribological areas within the considered piston pump the material combination is brass and hardened steel.

In order to determine the pump's health state (resp. degradation) continuously, the progression of the volumetric efficiency is monitored over time (refer to Figure 4). For a given operating condition the volumetric losses of a system are comparable [3]. This condition is determined by the temperature resp. viscosity, the operating pressure and the displacement rate.

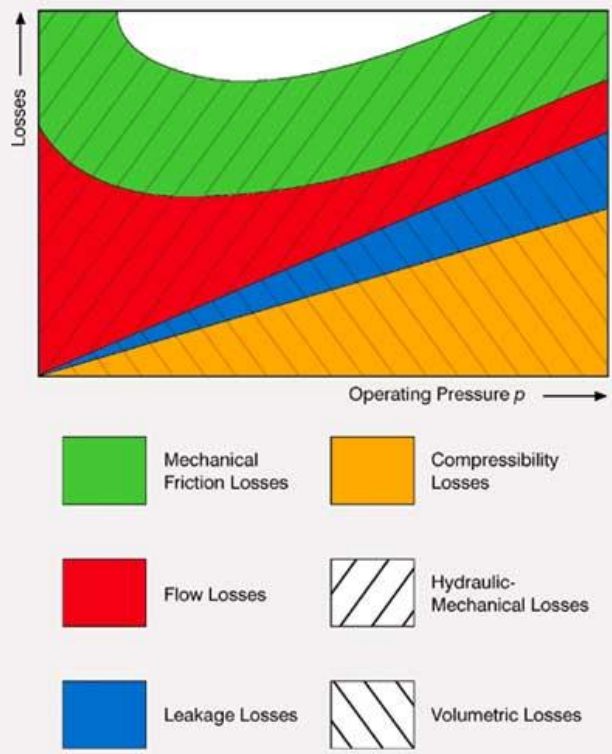

Figure 4: Losses in a hydraulic drive [3]

Accordingly, in order to determine the volumetric losses in the closed system at hand without a flow meter, the pressure holding speed is measured. This is the rotational speed of a fixed displacement pump, which is required to maintain a defined operating pressure at a given temperature. Depending on the level of degradation, an increase of the rotational speed is expected. In Figure 5 the progress of this speed is illustrated for the given EHA over its operating hours.

For the first 25000 operating hours the overall leakage is constant and the respective pump speed remains below 50rpm. In the following the continuous abrasion exceeds the filter capacity of the unit; the filter clogs and the increased pre- 
filter pressure causes the bypass valve to open. As a consequence the wear particles are circulating within the closed hydraulic circuit unfiltered. This results in exponential wear of the pump's sealing surfaces and leakage. A maximum value of 500rpm (factor 10) is reached after 27000 operating hours (refer to Figure 5).

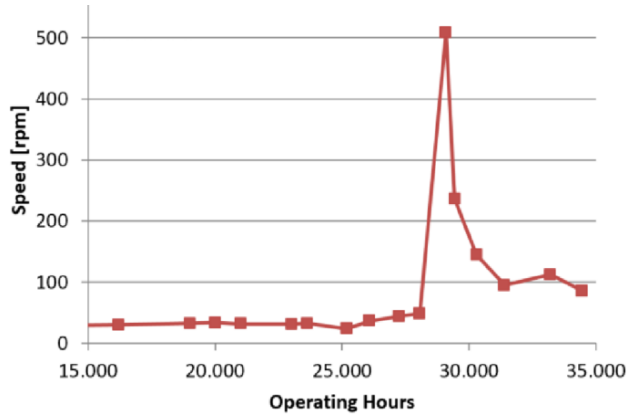

Figure 5: Progression of internal leakage over time for fluid temperature of $40^{\circ} \mathrm{C}$.

A subsequent inspection of the actuator indicated that the degrading performance is related to the contamination of the hydraulic fluid. An analysis of both fluid and filter revealed steel particles, which arise from the drive shaft bearings. The bearings showed wear marks, accordingly. The pump itself showed slight wear marks, but was considered to be undamaged still. Hence, the unit has been flushed with fresh oil and reassembled with a new set of bearings and filter cartridge for test continuation.

Once the unit has been maintained, the volumetric efficiency increased again (refer to Figure 5). Even though the pressure maintenance speed remains higher than the initial value, it stabilizes at about 100rpm, which is still within the nominal operating range of the actuator. This value is constant until the end of testing close to 35000 operating hours.

The fact that the performance of the unit recovers after the refresh of fluid, illustrates the failure tolerance of the EHA design.

However, while the pairing of cylinder and valve plate seems to be uncritical, for the given piston pump it was rather the slipper / swashplate interface, which suffered wear later on. Solely here material removal was visible; the slippers have been worn off dramatically to the end of the endurance test at about 35000 operating hours (refer to Figure 6). Not only was the material removed on the slipper sealing surface. As a consequence, from the increasing clearance between slipper, swash plate and retainer plate, shocks occurred between the slippers and the hardened retainer plate causing wear in the slipper shoulder.

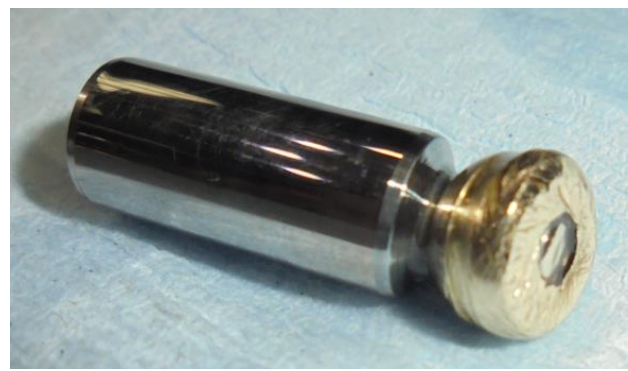

Figure 6: Slipper wear after 35'000 hours of endurance testing

Nevertheless, even though the wear of the slippers is immense, the EHA was still able to provide the required performance, when the first pistons have been separated from the slipper, so that they did not provide pressure anymore. This again illustrates the pump's failure tolerance.

However, the corresponding noise and vibration lead to the conclusion that condition monitoring is a reasonable approach to detect a pump failure and might possibly facilitate predictive maintenance.

Furthermore, this endurance test creates confidence in the pump technology and the specific EHA design. Several 10'000FH are more than what is required for today's aircraft, where the EHA technology is a backup solution that operates actively merely for 100 to $1^{\prime} 000 \mathrm{FH}$ within an aircraft life.

\subsection{Pump level tests}

An even more efficient approach to investigate the pump wear behavior are tests on pump level. While a flight control EHA is a highly integrated and complex unit, the Liebherr-Aerospace pump test rig (refer to Figure 7) provides a deeper view inside the pump via additional laboratory sensors, like particle sensors and viscometers as well as a number of temperature sensors. Following the endurance spectrum of the considered actuator, sinusoidal oscillations are applied covering a representative range and amount of pressure and speed cycles (refer to section 1). 


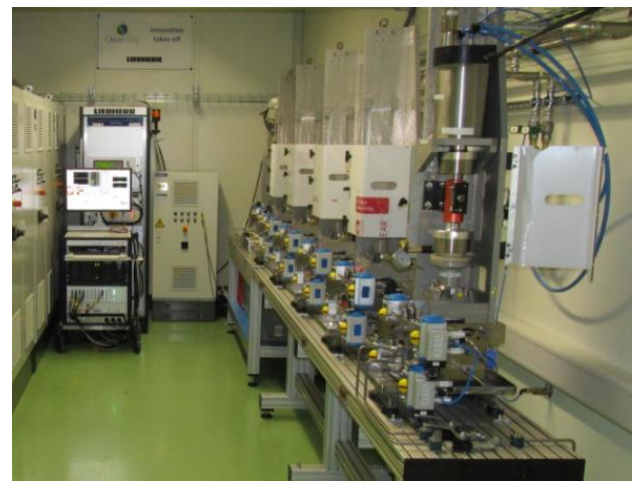

Figure 7: Pump level test rig at Liebherr-Aerospace Lindenberg $\mathrm{GmbH}$

Thereby the influence of distinctive operating conditions (speed, pressure, temperature, fluid etc.) on the generation of wear particles can be investigated. The hydraulic schematic of the pump test rig is illustrated in Figure 8.

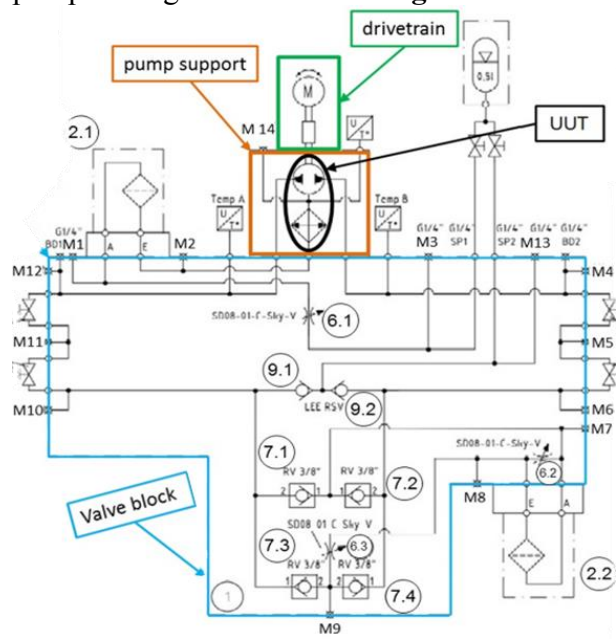

Figure 8: Pump level test (schematic)

Another purpose of the pump test rig is to reproduce the wear mechanism that has been identified during the previous unit level tests and thereby validate the test results. Furthermore, condition monitoring functions, which have been developed model based, are validated iteratively within the pump test rig environment. Finally, the goal is to facilitate and expedite the generation of reliable data that allows for the optimization of the pump design or the system architecture (choice of fluid) as well as for the online monitoring of its health condition.

Initially, the basic characteristics of the pump have been determined. In order to measure the drag torque in different operating points a torque measuring shaft was introduced into the drive train. Subsequently the data was post-processed, in order to distinguish between the load and the relevant drag torque as follows:

$T_{\text {drag }}=T-\frac{\Delta p \cdot D}{2 \pi}$

In Figure 9 the drag torque is illustrated for various pressure and temperature levels. Similar to the Stribeck curve dry friction occurs for low rotational speed. In case of lubricated friction at higher speed, the drag torque is reduced to a minimum at around 500rpm. For even higher speed of several 1000rpm viscous fluid friction is dominating. However, in contrast to the static friction at low speed, solid contact is avoided.

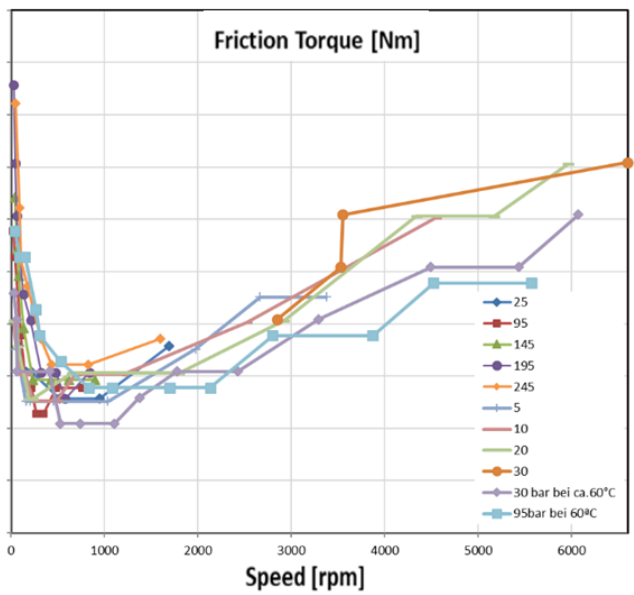

Figure 9: Calculated drag torque for varying speed and pressure level

However, it is remarkable that the drag torque is independent from the pressure level, which has been varied between 5 bar and 245bar. Furthermore, the reduced viscosity at higher temperatures (violet and light blue line) results merely in a slight increase of low speed friction, but also in a reduced fluid friction at high speed.

Primary flight control actuators (e.g. aileron) perform an oscillating (corrective) motion during flight, which causes the pump to be operated bidirectionally. According to Figure 9 every change of direction is connected to a start-stop, where lubrication is a key. 
The unit level tests highlighted the major importance of the lubricant for the health condition of the EHA. Hence, on pump level the fluid condition is monitored by several means.

First, the particle contamination is identified with the aid of particle sensors according to the SAE AS4059. Depending on the particle size (2$25 \mu \mathrm{m})$ the respective class of contamination is illustrated in Figure $\mathbf{1 0}$ over time. While the sensor does not distinguish between the type of particles, the initial fluid contamination is high. Due to the integrated filter $(5 \mu \mathrm{m})$ the amount of particles in class B, C and D is reduced throughout the testing $(>400 \mathrm{~h})$. With continuing abrasion the filter capacity is exceeded after $900 \mathrm{~h}$ of testing. Here the fluid contamination increase rapidly, which is correlating with an increased pre-filter pressure.

Particle Classification (SAE AS4059)

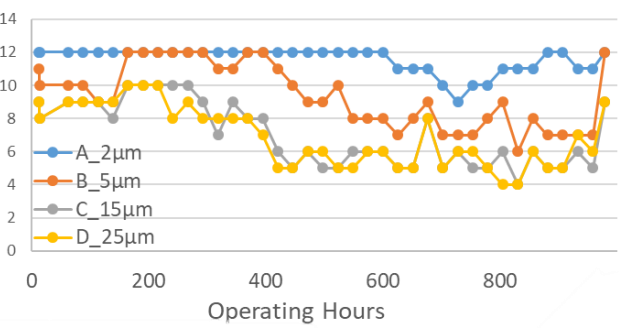

Figure 10: Generation of fluid contamination

As mentioned above, the particle sensor does not distinguish between metallic and other sources of contamination, like dust. However, especially the hard metallic materials are more harmful for the pump wear. Further, particle sensors do not detect micro-particles $(<2 \mu \mathrm{m})$. Yet, it is considered that the amount of micro-particles increase, whereas greater particles are bound within the filter.

In contrast, the fluid conductivity constitutes a more expressive characteristic (refer to Figure 11). Precisely because it does not differ the size of the particles, it relates to the overall amount of metallic contamination within the system; and therefore it is a significant characteristic with respect to the progressing deterioration due to tribological wear. In contrast to the complex particle sensors, the determination of the fluid conductivity is less complex and therefore more reliable.

The sensitivity of the conductivity is illustrated by the curve in Figure 11. It has a slightly exponential characteristic and spans over magnitudes. Similar to the particle concentration (refer to Figure 10), the conductivity also increases rapidly after $900 \mathrm{~h}$, when the filter clogs. However, the fact that the first conductivity measurement after 13 hours of operation is merely $1.5 \mathrm{nS} / \mathrm{m}$ points out that the initial fluid contamination given by the particle sensor is not resulting from metallic but rather dust particles.

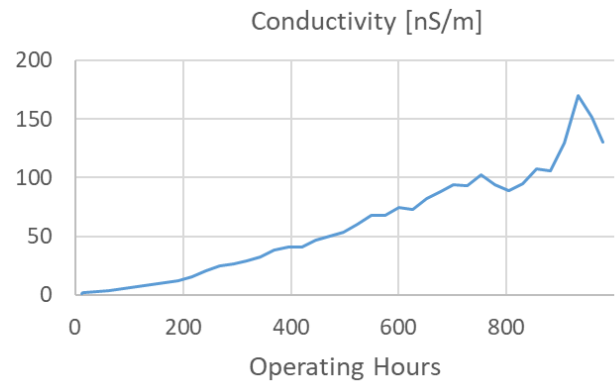

Figure 11: Generation of fluid conductivity

\section{CURRENT PUMP DESIGN}

Within the previous section test data was presented, which illustrates both the robustness of the current Liebherr piston pump and the wear effects related to the primary flight control application.

In the following, the current pump design is described in detail with respect to the wear findings. Design improvements are presented, which have potential of optimization with respect to long-life operation of the pump.

\subsection{Operating conditions}

In order to increase the power density and hence to minimize the weight, the pump used in Liebherr-Aerospace EHA has a displacement less than $1 \mathrm{~cm}^{3} / \mathrm{rev}$ and operates on a very broad range of velocity.

In addition to that, the design of the EHA is very compact, see Figure 1, and it is typically encapsulated within the aircraft structure. According to [4], in spite of counter-measures at the aircraft level, for instance with openings made in the wings to improve air circulation and cooling, the heat generation within the EHA is a critical issue.

Due to these severe operating conditions, designing an EHA pump with high performance and long service time is a challenging task, which can be broken down into four main issues, 
according to [5]:

- Cavitation erosion, mainly a consequence of high rotation speed and high pressure necessary for achieving a good power density

- Flow and pressure ripples, because of the difficulty to optimize a valve plate of EHA pumps on a very broad operating range

- Tilting motion of the rotating group, again a consequence of high rotation speed leading to high centrifugal forces of pistons and slippers

- Heat issue, because of high power density and compactness

\subsection{Inclined-piston design}

The pump under investigation, developed by Liebherr, is an axial-piston pump of swash plate type whose 7 pistons are inclined with respect to the rotation axis of the pump, see Figure 12.

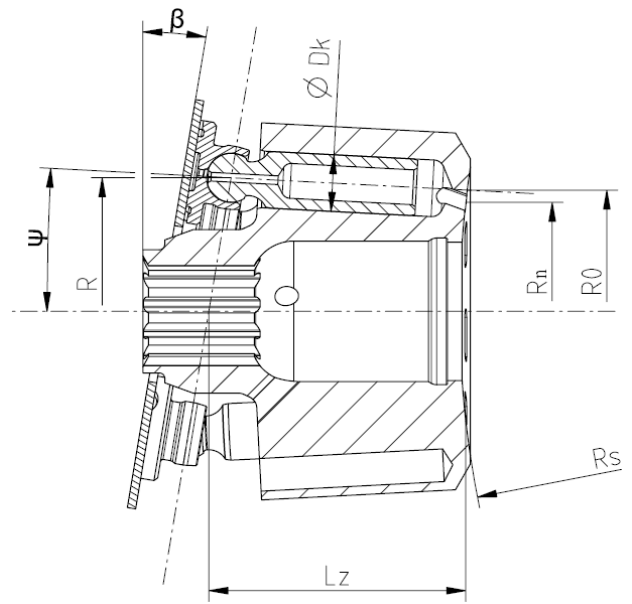

Figure 12: Sketch of the rotating group with pistons inclined with respect to rotation axis

The piston stroke for such a pump is given in [6] as follows:

$H_{K}=\frac{2 \cdot R \cdot \tan \beta}{\cos \psi \cdot(1-\tan \psi \cdot \tan \beta)}$

Where:

- $\quad \beta$ is the swash plate angle

- $\psi$ is the inclination angle between the pistons and the rotation axis of the pump

- $R$ is the distance from the piston sphere center to the rotation axis of the pump when the piston is at the inner dead center (furthest position within the cylinder-block)
Axial-piston pumps with inclined pistons are less common than in-line pumps and show more complicated kinematics and dynamics. Nevertheless they have a few very interesting characteristics, especially for high-speed applications.

\subsection{Clamping force on the slippers}

At high rotational speed, two main effects tend to separate the slippers from the swash plate. The first significant effect arises because the center of mass of the slipper does not generally coincide with the center of the piston-slipper ball joint. Therefore, the centrifugal forces acting on the slippers generates a centrifugal tilting moment that tend to tilt the slipper towards its inner edge. For inclined-piston designs like the pump under investigation, this effect is slightly higher near the outer dead center where the slipper is further from the pump rotation axis.

The second - even more - significant effect is due to the axial acceleration of piston-slipper assemblies. The resulting inertial force pushes the slipper against the swash plate during $180^{\circ}$ of its travel on the outer dead center side while it tends to pull the slippers away from the swash plate during the other $180^{\circ}$ on the inner dead center side.

In the region of the inner dead center, the two main effects described above add to each other and a sufficient clamping force is needed to prevent the slippers from lifting or tilting. In pumping mode, when the slipper approaches the inner dead center, it is connected to high-pressure port and a sufficient clamping force can be achieved with available pressure. However, once the slipper has passed over and goes away from the inner dead center, it is connected to the lowpressure port, hence with only a limited clamping force available from the pressure. The situation is even more critical if one considers that due to pressure losses in the inlet duct at high speed, the actual pressure in the piston chamber might be even lower than the inlet pressure.

Therefore, sufficient clamping of the slippers requires countermeasures to be taken like for instance increasing the inlet pressure and/or using a positive force retaining mechanism. However both these design changes induce extra losses, especially at low speed where this extra clamping force is actually not needed.

In this context, the design of the Liebherr EHA 
pump has the benefit that the inclined pistons generate an axial component of the centrifugal force that act as an additional clamping force on the slippers. Since this force increases with the square of the rotational speed, it is mainly present at high speeds and does not cause additional losses at low speeds. For the pump under investigation, at high speed the inclined pistons generate a significant additional clamping force that is equivalent to an increase of approx. $2.5 \mathrm{bar}$ in the inlet pressure.

\subsection{Tilting of the cylinder-block}

When an axial-piston pump operates at high pressure, the pressure in the cylinder bores typically generates a clamping force that helps maintaining the cylinder-block against the valve plate. However when the pump operates at low pressure and high speed, the inertial forces transferred from the pistons to the cylinder block tend to destabilize it by generating a tilting moment and since no clamping force is available from the pressure it is necessary to axially preload the cylinder block with the help of a spring. Again, this additional axial preload of the cylinder induces undesired extra losses, especially at low rotational speeds when this preload is not required.

Another interesting effect of the inclined pistons used in the Liebherr EHA pump is that the inertial forces acting on the piston/slipper assemblies generate an additional clamping force pushing the cylinder block against the valve plate. As for slippers, the clamping force generated on the cylinder block is only significant at high speeds and does not cause any loss at low speeds. For the pump under investigation, the inclined pistons allowed to reduce by a factor 2.0 the theoretical spring preload needed to prevent the cylinder block from tilting at high speed in comparison to a design with in-line pistons.

\section{PUMP DESIGN IMPROVEMENTS}

\subsection{Slipper / swash plate interface}

The test results presented in this article show that the slipper / swash plate interface has suffered dramatic wear after $35^{\prime} 000 \mathrm{FH}$, which is an indication that there is room for optimization of the lubrication of this sliding interface. The lubrication mechanism involves a complex thermal-fluid-structure interaction that has been addressed by an impressive research effort conducted in the recent years, especially under the guidance of Prof. Ivantysynova at Purdue University, see for instance [7]. Nevertheless, the simple macroscopic approach of computing a socalled hydrostatic balance factor is still of value when comparing two designs. The balance factor of a slipper in an inclined-piston design is very seldom detailed in literature. It is obtained as the ratio of the force generated by the slipper hydrostatic bearing $F_{\text {hydro }}[N]$ to the total reaction force perpendicular to the swash plate in static equilibrium condition $F_{\text {reac }}[N]$.

The hydrostatic bearing force is the same for in-line and inclined-piston design. It is obtained as follows:

$F_{\text {hydro }}=\frac{\pi}{8} \cdot \frac{D_{G a}^{2}-D_{G e}^{2}}{\ln \left(\frac{D_{G a}}{D_{G e}}\right)} \cdot P_{H D}$

However, the total reaction force perpendicular to the swash plate surface is specific to the inclinedpiston design:

$$
F_{\text {reac }}=\frac{\pi \cdot D_{K}^{2} \cdot P_{H D}}{4 \cdot\left(\begin{array}{c}
\cos \beta \cdot \cos \psi \\
-\sin \beta \cdot \sin \psi \cdot \cos \phi_{i}
\end{array}\right)}
$$

Where $\phi_{i}$ is the angular displacement of the considered $\mathrm{i}^{\text {th }}$ piston (with $\phi_{i}=0^{\circ}$ at the outer dead center) and the diameters $D_{K}, D_{G a}$ and $D_{G e}$ are shown in Figure 13.

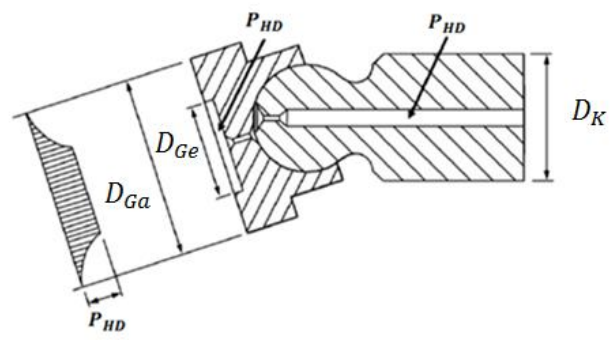

Figure 13: Hydrostatic pressure field under the slipper

It follows that the hydrostatic balance factor is slightly higher at inner dead center than at outer dead center. For the pump under investigation, this difference is, however limited to less than $2 \%$ and the balance factor of the slipper at inner dead center reaches a maximum value of approx. $90 \%$. This indicates that a better lubrication - yet without significant increase of leakage - can be 
achieved by increasing this value compared to the tested pump.

\subsection{Valve plate design}

The valve plate is a key component in an axial piston pump. Among others, it affects particularly the cavitation and the pump solid borne noise because it controls the pressure transients in the piston chambers. The pump under investigation does not feature any pressure relief grooves in the transition regions but rather has fixed pre- and decompression angles. While this design can give optimal results for one given operating point (working pressure and speed) this may lead to increased cavitation and/or extremely fast pressure transients for other operating points.

Therefore, the original design of the valve plate has been reviewed and a new valve plate has been proposed with pressure relief grooves. This new design aims at smoothing the pressure transitions over a relatively broad operating range. The numerical simulations conducted are based on a classical control volume approach described for instance in [8]. Example results for the obtained smoothing of pressure compression are shown in Figure 14 and in Figure 15.

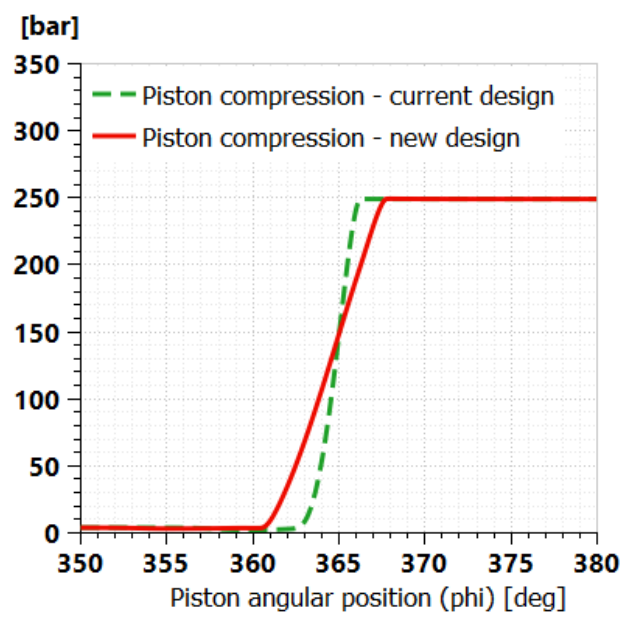

Figure 14: Simulated pressure transients in piston chamber at outer dead center

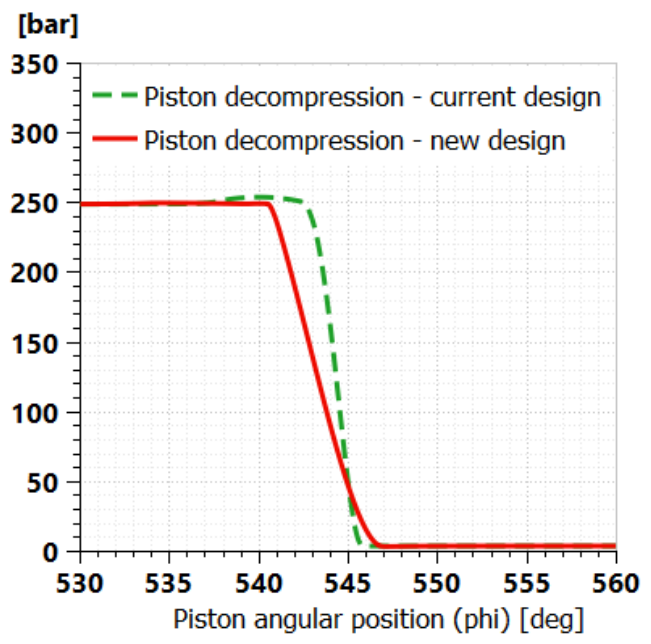

Figure 15: Simulated pressure transients in piston chamber at inner dead center

\section{CONCLUSION AND OUTLOOK}

In general, this paper illustrates Liebherr's experience regarding piston pumps in primary flight control actuators, respectively EHA. The variety of data gathered by testing activities proves the robustness and failure tolerance of the current pump design. It complies with the challenging requirements of future more electric aircraft architectures. Therein the EHA could replace the conventional servo-hydraulic actuation and be operated in frontline for the whole aircraft life.

However, the test campaign also revealed the wear mechanism, which ultimately causes the piston pump to fail. Accordingly, two possible design modifications have been presented that might further increase the life time of the EHA technology. In particular the adaption of the hydrostatic balance factor of the slipper / swash plate interface is considered to be beneficial.

With the aid of the presented pump level test rig it is then possible to perform comparative test, in order to validate optimized pump designs. Moreover, innovative material combinations will be investigated. Apart from their wear behavior, hereby focus is also on materials compliant to environmental legislation, with a reduced share of lead for instance. 
Furthermore, the acquired test data indicates, that despite the general robustness of the Liebherr pump design, a degradation of performance sets in, if the fluid quality cannot be assured. In case of a contamination with metallic particles the internal leakage within the system can increase exponentially. Hence, the continuous filtration of the hydraulic system is the key for long-term operation of EHA in primary flight controls.

Nevertheless, a degradation takes place over a considerably long time span and can be identified based on the monitoring of some basic features. The benefit of condition monitoring functions has been highlighted as a measure to reduce the remaining technological and financial risk constituted by today's limited maturity of EHA in civil aviation.

\section{REFERENCES}

[1] Roman Ivantysyn (2018) Investigation of the Thermal Behavior in the Lubricating Gap of an Axial Piston Pump with Respect to Lifetime, IFK2018, Aachen, Germany

[2] Gertraud Wolff (1977) Lässigkeitsverluste in Axialkolbenpumpen, Agrartechnik, Heft 5, 225 227
[3] Brendan Casey (2004) Determining Hydraulic Pump Condition Using Volumetric Efficiency, Machinery Lubrication, 9/2004

[4] Maré J-C (2017) Aerospace Actuators Vol. 2: Signal-by-Wire and Power-by-Wire, ISTE Editions Ltd., London

[5] Chao Q, Zhang J et al (2016) A Review of HighSpeed Electro-Hydrostatic Actuator Pumps in Aerospace Applications: Challenges and Solutions, J of Mechanical Design, Vol. 141

[6] Ivantysyn J, Ivantysynova, M. (2001) Hydrostatic Pumps and Motors: Principles, Design, Performance, Modelling, Analysis, Control and Testing, Academia Books, Delhi

[7] Schenk A, Ivantysynova M (2011) An Investigation of the Impact of Elastohydrodynamic Deformation on Power Loss in the Slipper Swashplate Interface. In: Proceedings of the 8th JFPS International Symposium on Fluid Power, Okinawa, 2011

[8] Viennet E, Gaile A, Roeben T (2018) Noise and vibration reduction for an aerospace secondary controlled hydraulic motor, 11th International Fluid Power Conference, Aachen, 2018 